\title{
Disruptive insights in psychiatry: transforming a clinical discipline
}

\author{
Thomas R. Insel
}

National Institute of Mental Health, NIH, Bethesda, Maryland, USA.

\begin{abstract}
Mental disorders such as schizophrenia, bipolar illness, and depression have become the predominant chronic diseases of young people, accounting for approximately $40 \%$ of the medical burden for people aged 15-44 in the United States and Canada. Research is transforming our understanding of these disorders, as exemplified in the articles in this Review Series. Important, "disruptive" insights into pathophysiology are emerging from studies addressing these illnesses as brain disorders, developmental disorders, and complex genetic disorders - rather than only as psychological conflicts or chemical imbalances, as they were considered in the past. Current medications are not sufficient for most patients. A new and deep understanding of the pathophysiology of these disabling disorders is our best hope for a new generation of treatments that will help patients to recover.
\end{abstract}

\section{Introduction}

Much of what I learned as a resident in psychiatry 30 years ago has been proven utterly wrong. At that time, we believed that peptic ulcer disease was due to intrapsychic conflicts treatable with psychoanalysis; autism resulted from rigid, cold mothers who drove their children into psychic isolation; and schizophrenia could be treated through a regressive process allowing the patient to "work through" the psychosis as a growth experience. Now, these and other myths have been discarded as a result of scientific research. Perhaps our successors 30 years from now will look back on much of what we believe today with the same rueful perspective. The challenge, of course, is identifying those elements of current theory and practice that need to be left behind. This Review Series, "Rethinking Mental Disorders," is dedicated to some of the emerging areas of discovery that may overturn many of our current beliefs about schizophrenia (1), depression (2), bipolar illness (3), childhood-onset obsessive-compulsive disorder (OCD) (4), and autism spectrum disorder (ASD) (5). As an introduction to the individual Review articles, I set the stage by identifying some of the conceptual shifts, or, as I refer to them here, "disruptive insights," that have come about through current research. But first, we should consider the public health significance of mental disorders.

\section{Public health significance of mental disorders}

According to the WHO, mental disorders are the number one source of medical disability for people 15-44 years of age in the United States and Canada, accounting for nearly $40 \%$ of all medical disability in this age range (6). Several mental disorders are highly prevalent; for example, $16 \%$ of the population will experience major depressive disorder (which is characterized by persistent and disabling sense of sadness, emptiness, hopelessness, helplessness, and worthlessness) at some point in their lifetime, and $6.6 \%$ of the population is affected by this disorder each year (7). Major depressive disorder can be a crippling mood disorder, with

Conflict of interest: The author has declared that no conflict of interest exists.

Nonstandard abbreviations used: ADHD, attention-deficit/hyperactivity disorder; ASD, autism spectrum disorder; ASHD, arteriosclerotic heart disease; GWAS, genome-wide association study; OCD, obsessive-compulsive disorder.

Citation for this article: J. Clin. Invest. 119:700-705 (2009). doi:10.1172/JCI38832. patients unable to work or function during an episode. In contrast to many other disabling, chronic illnesses, mental disorders begin early in life. There are, of course, mental disorders that we associate with childhood, such as attention-deficit/hyperactivity disorder (ADHD) and ASD. But even for so-called adult illnesses, such as mood and anxiety disorders, $50 \%$ of patients describe onset by age 14 (8). Indeed, in the developed world, mental and addictive disorders are increasingly the chronic disorders of young people.

Mental disorders are a major source of not only morbidity but mortality. In 2005 (the most recent year for which official mortality statistics are available), there were more than 32,000 suicides in the United States, $90 \%$ of which were believed to be associated with mental illness (9). This is nearly twice the number of homicides and greater than the number of deaths from AIDS (10). Beyond mortality from suicide, people with serious mental illness (e.g., schizophrenia and bipolar disorder) die early, with an average life expectancy of 56.3 years (11). This early mortality is believed to be due predominantly to the medical complications of these illnesses or their treatment. Tobacco use also may be an important mediator of increased mortality in individuals with mental illness. While approximately $26 \%$ of the population experience mental illness in a given year, Lasser et al. estimate that $44 \%$ of cigarettes are consumed by individuals with current mental illness (12).

Most mental disorders are treatable with medication or psychosocial interventions. Effective antidepressants, anxiolytics, and antipsychotics have been available for more than four decades. Second-generation compounds, available for the past decade or more, are not demonstrably more efficacious but have either fewer or different side effects $(13,14)$. The availability of effective medications for these syndromes has been both a blessing and a curse. Clinicians have been able to reduce the hallucinations and delusions of psychosis and to relieve the hopelessness and helplessness of depression in many patients. But the high levels of morbidity and mortality described above demonstrate that current medications are not sufficient. The focus on medications has shifted resources away from psychosocial treatments such as structured, short-term behavioral therapies that are effective for some mood and anxiety disorders (15) and rehabilitative services that improve functional outcomes for people with schizophrenia. For too many people, antipsychotics and antidepressants are not effective, and even when they are helpful, they reduce symptoms without eliciting recovery. 
A Spread of cortical maturation

ADHD

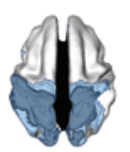

7 years

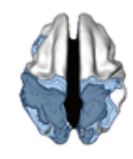

8

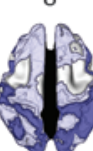

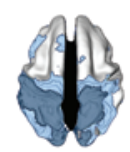

9

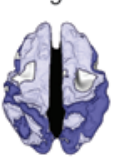

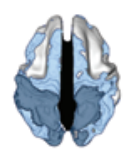

10

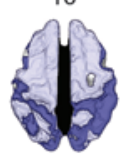

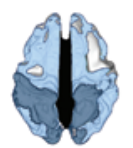

11

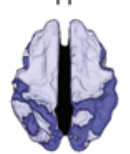

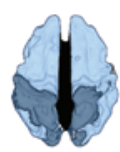

12

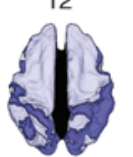

B Age of attaining peak cortical thickness

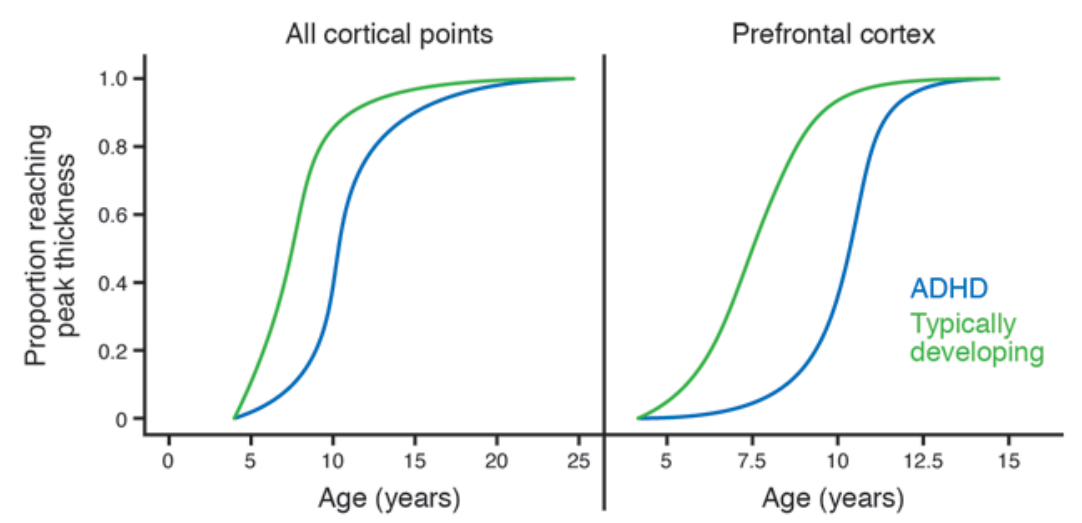

\section{Figure 1}

The development of cortical thickness in children with ADHD is markedly delayed in comparison with that in control children who are developing typically. (A) Dorsal view of the cortical regions where peak thickness was attained at each age. The darker colors indicate regions where a peak age could not be calculated or the peak age was estimated to lie outside the age range covered. The sequence in which the regions attained peak thickness was similar in the two groups, but the children with ADHD showed considerable delay in reaching this developmental marker. (B) Kaplan-Meier curves illustrating the proportion of cortical points that had attained peak thickness at each age for all cerebral cortical points or the prefrontal cortex. The median age by which $50 \%$ of cortical points had attained their peak differed significantly between the groups (all $P<1.0 \times 10^{-20}$ ). Adapted with permission from Proceedings of the National Academy of Sciences (24).
Approximately $80 \%$ of individuals with schizophrenia are unemployed, and more than half of those with bipolar disorder also experience problems with substance abuse or addiction $(16,17)$. This brings us to the curse. Most biological research in psychiatry has been focused on understanding how psychiatric medications work. If the medications were curative, this might have been revealing. But these medications, at best, ameliorate symptoms, and when they are effective, the effects usually require weeks of treatment. Indeed, the pharmacological effects have an unknown relationship to the pathophysiology of the mental disorders. In the past four decades, we have learned quite a bit about the mechanisms of antipsychotic and antidepressant drug action but surprisingly little about the pathophysiology of psychosis and mood regulation.

Clearly, before we can develop the next generation of medications, we need a deeper understanding of the pathophysiology of mental disorders. The good news is that we now have the tools to enable a new science of mental disorders. I believe that this science will revolutionize the diagnosis and treatment of mental illness and that it will transform psychiatry, ultimately realigning it with neurology and potentially creating a new discipline of clinical neuroscience (18). The present series, "Rethinking Mental Disorders," introduces several of the front lines of this revolution. It may be notable that this revolution is infused by new ideas from outside psychiatry: the authors include a neurologist (2), a developmental neuroscientist (5), and a pediatrician (4). In this overview, I describe four conceptual shifts, or disruptive insights, that provide the intellectual basis of this revolution.

\section{Mental disorders are brain disorders}

By Congressional proclamation, the 1990s were designated the "Decade of the Brain" (19). Beyond bringing public attention to the excitement of neuroscience research, that decade marked an end to a three-century division between mind and brain. Increasingly, the most complex aspects of the mind were being addressed through studies of neural activity. The advent of structural and functional neuroimaging, as well as more sophisticated behavioral neurophysiologic research, began to shed light on previously inaccessible mental events, such as decision making, moral judgments, and consciousness.

In the current decade, the focus on mental activity as neural activity has widened to include a focus on mental disorders as neural disorders. Previous biological hypotheses posited that depression and schizophrenia were due to a chemical imbalance, as if an overall deficit in serotonin or increase in dopamine could explain these illnesses. Neuroimaging helped to open up the black box of the brain so that these disorders, for the first time, could be studied as disorders of neural systems and not simply as a lack of the requisite medication or an altered amount of a single neurotransmitter. Recent results are encouraging, as described in several of the articles in this Review Series (1-3). For one thing, all the mental disorders studied thus far using neuroimaging techniques involve the prefrontal cortex. Aspects of schizophrenia can be mapped onto dysfunction of dorsolateral prefrontal circuits that mediate executive function (i.e., functions such as judgment, planning, and cognitive flexibility) (20); depression appears to involve dysfunction in a heretofore unexplored region of the midline infragenual prefrontal cortex that is important for regulation of mood (21); OCD involves dysfunction in the orbitofrontal prefrontal cortex via its role in perseverative behaviors (22); and posttraumatic stress disorder (PTSD) can now be mapped in a precise way to dysfunction in prefrontal circuits required for the extinction of fear (23). Just as important as the apparent localization is the distribution 


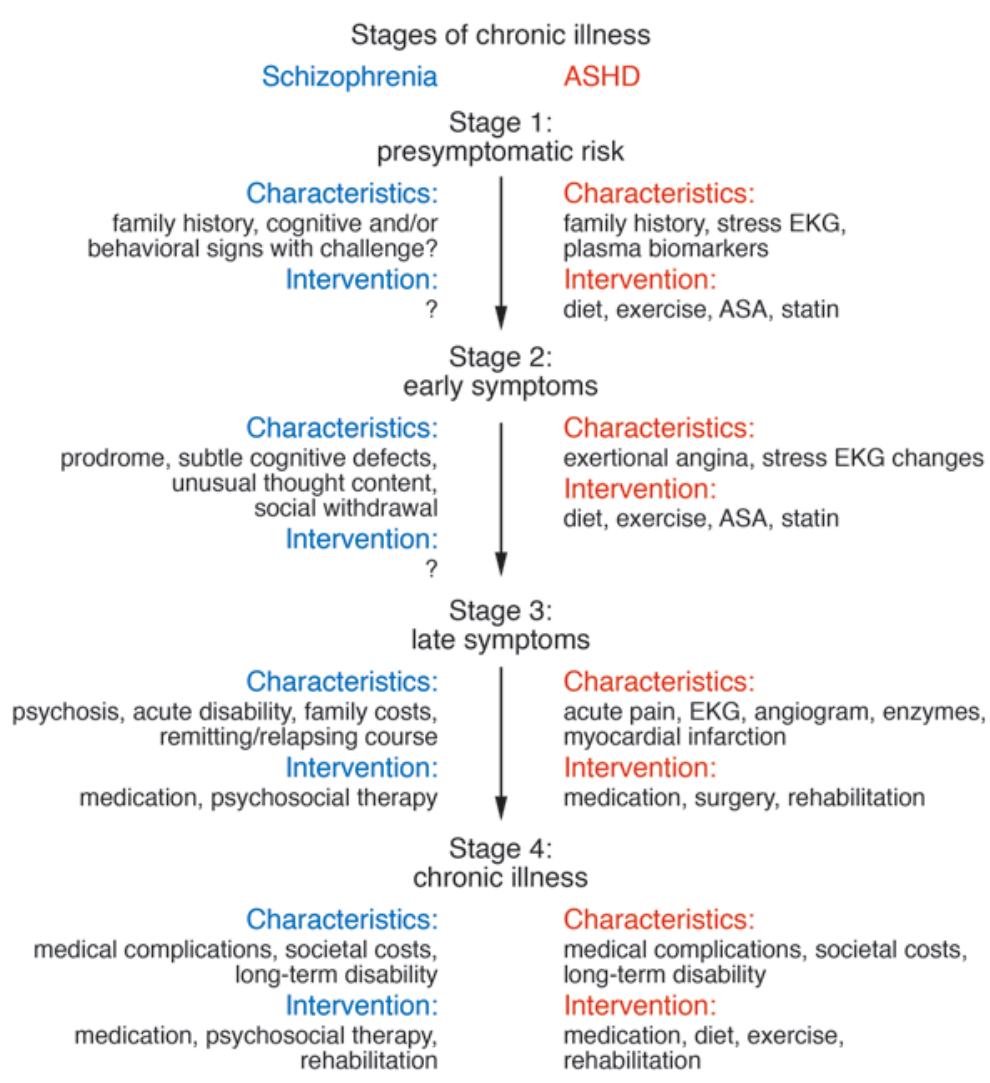

\section{Figure 2}

Rethinking schizophrenia: identifying the illness before onset of psychosis. In the future, schizophrenia may be viewed as a multistage disorder with psychosis analogous to myocardial infarction in ASHD. As in ASHD, early detection could introduce an era of preemptive treatments for schizophrenia, yielding better outcomes by halting the disease process before onset of psychosis. ASA, acetylsalicylic acid.

brain disorders may help to reduce stigma. Many individuals still do not accept mental disorders (including ADHD) as real illnesses. If the general population understood ADHD as a disorder of cortical maturation, one would hope that there would be less shame and blame directed at the families who struggle with these illnesses.

Of course, we need to avoid the oversimplified approaches that have traditionally characterized biological and psychological hypotheses about these illnesses. These illnesses result from complex biological and psychological processes. Importantly, the biological (neural) substrates for these disorders may precede the psychological manifestations. We know from studies of neurodegenerative diseases, from Alzheimer disease to Parkinson disease, that the observable behavioral manifestations are late events in the course of the diseases (25). Similarly, in mental disorders, whether we are focused on hyper-

of the pathological activity. If most neurological disorders, from stroke to Parkinson disease, can be conceptualized as focal brain lesions, many psychiatric disorders can be understood as dysfunctions within brain circuits. As an imperfect metaphor, consider the difference between myocardial infarction, with focal loss of cells, and a severe conduction defect, which alters myocardial function without focal cell loss. Both result in severe dysfunction, but they involve different forms of pathology.

It is easy to underestimate the significance of this disruptive insight. If mental disorders are brain disorders causing psychological distress rather than psychological disorders caused by psychic trauma or conflict, then our approaches to diagnosis, treatment, and professional training need to be rethought. Recent research in ADHD affords a useful example. Shaw et al. reported a prospective, longitudinal MRI study of 223 children with ADHD and 223 unaffected controls (24). Mapping the trajectory of the development of cortical thickness in the two groups demonstrated that the ADHD children were delayed by about three years in the pattern of cortical maturation, with the greatest delays (of about five years) apparent in the middle prefrontal cortex (Figure 1). Across the entire cerebrum, the median age for attaining peak cortical thickness was 10.5 years in the ADHD children versus 7.5 years in the controls (24). Here is a disorder defined by behavioral and cognitive features (hyperactivity and attention deficit, respectively) that appears to be a disorder of cortical maturation. Again, to use the cardiovascular metaphor: it is similar to diagnosing myocardial infarction as a chest pain disorder, without any reference to the underlying mechanisms of the symptoms.

In addition to forcing us to reformulate diagnosis, treatment, and training, the recognition of mental disorders as activity, psychosis, or depression, it is possible that these behavioral manifestations represent a late stage of abnormal neural system activity. This brings us to our next disruptive insight.

\section{Mental disorders are developmental disorders}

As noted above, mental disorders can be considered the primary chronic diseases of young people in this country. This sets mental disorders apart from many other brain illnesses that are degenerative and begin late in life. The onset of mental illness generally occurs early in childhood or adolescence, even though diagnosis may be delayed for years if not decades. Wang et al. report that for mood disorders, the mean delay is 6-8 years; for anxiety disorders, 9-23 years, depending on the disorder (26). Some of this delay may be explained by the fact that the manifestations at the early phases of these disorders are different from those of their later forms. Depression in adulthood may be preceded by anxiety disorders in childhood (27-29). A schizophrenic psychosis at age 18 is often preceded by a prodromal phase, a period of early signs and symptoms that can be identified 2-3 years before onset of psychosis (30). Further, as children at risk for ASD have been studied more carefully, early forms of this disorder have been detected, appearing long before speech delay or social deficits (31). Understanding the developmental stages of these disorders will be transformative. Currently, schizophrenia is defined by psychosis, just as arteriosclerotic heart disease (ASHD) was once defined by myocardial infarction. Increasingly, we are beginning to view psychosis as a but even earlier by many years of risk, analogous to the stages of risk and ischemic changes of ASHD. The identification of these pre-psychosis stages suggests the opportunity for earlier diagnosis late stage of schizophrenia, preceded not only by the prodrome 


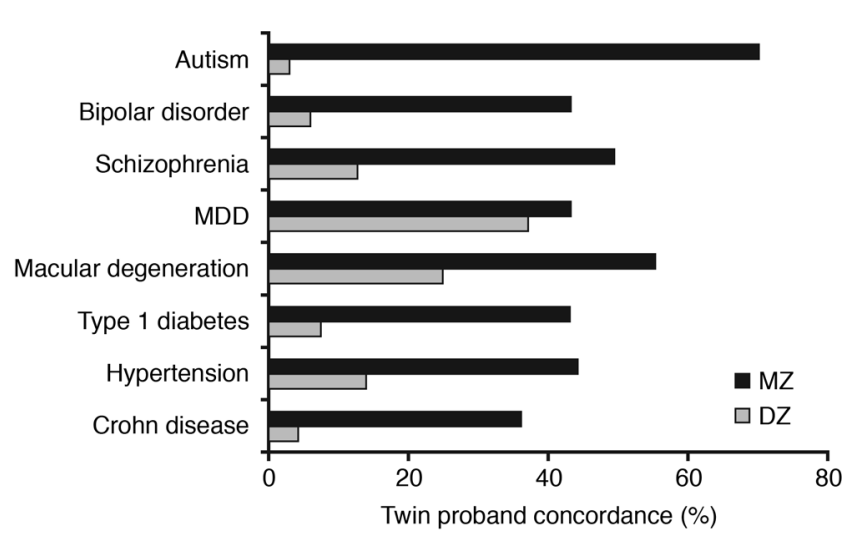

(Figure 2). Even more important than early detection, understanding mental disorders as developmental disorders suggests that we may be able to intervene earlier and preempt the later, disabling stages of these illnesses. This requires some explanation.

In Parkinson disease, we know that the observable symptoms emerge only after $80 \%$ of the dopamine cells in the substantia nigra deteriorate. In amyotrophic lateral sclerosis (ALS), motor weakness becomes evident with the loss of virtually all the requisite motor neurons $(32,33)$. It may be a general rule that behavioral and cognitive symptoms are late manifestations of underlying neuropathology. In the case of mental disorders, if the underlying pathology is a disordered circuit (as opposed to loss of a focal group of cells), the observable symptoms may manifest only after the circuit fails to develop functional connections, after the circuit is required in development, or after a compensatory circuit can no longer suffice. Whatever the trigger for symptoms, the underlying pathophysiology may be apparent long before behavioral and cognitive symptoms emerge. Research can now be directed at identifying biomarkers that detect risk and/or early stages of these disorders and developing interventions that preempt the later stages. Just as we use plasma lipids and cardiac imaging to identify early stages of ASHD, we need biomarkers for identifying schizophrenia or bipolar illness long before the onset of psychosis. Developing the "statins" that will preempt the psychosis of schizophrenia or bipolar illness may well be the next frontier for psychiatric therapeutics and could be the most promising area for reducing the morbidity and mortality of these disorders.

\section{Mental disorders are complex genetic disorders}

By almost any estimate, the past two years have seen a revolution in clinical genetics. Genome-wide association studies (GWASs) have revealed much of the genetic risk architecture for many common diseases, including adult-onset macular degeneration (34), inflammatory bowel disease (35), and type 2 diabetes mellitus (36-38). In each case, while the risk conferred by individual associations appears small, the findings collectively have provided important new insights into pathophysiology. How have GWASs informed the pathophysiology of mental disorders? The heritabilities of ASD, bipolar disorder, and schizophrenia appear higher than for many of the common disorders with large GWAS findings (Figure 3); however, there have been relatively few substantiated GWAS findings for mental disorders thus far. Although several studies have been published, either the size of the cohort or the heterogeneity of the diagnostic category appears to have precluded finding significant associations (39-41).

\section{Figure 3}

Estimated monozygotic (MZ) and dizygotic (DZ) twin concordance rates for various neuropsychiatric and other medical disorders. Data from twin studies indicate that the heritabilities of mental disorders are higher those of many common disorders that have yielded significant findings in GWASs. MDD, major depression disorder. Compiled from refs. 57-64.

Nevertheless, neuropsychiatric genetics is making important strides, with many significant candidate genes identified for each of the major disorders, as discussed for ASD in the article in this Review Series by Pat Levitt and Daniel Campbell (5). In neuropsychiatric genetics, as in neuroscience, the emerging science is providing some disruptive insights. One is the importance of DNA structural variation, including increased and decreased copy numbers of broad stretches of DNA, in patients with ASD and schizophrenia (42-44). Some of these structural changes are de novo, presumably explaining the continued high prevalence of these disorders despite low reproductive rates (45). While we know relatively little about the functional significance of structural variation, this discovery reminds us that SNPs, which have been the staple of psychiatric genetics, are only one of many forms of variation in the genome. Another important insight is genomic heterogeneity, even in patients carefully selected for similar clinical features. Clearly, there are many different forms of genomic risk for each of these disorders. This complicates the search for a large-effect gene, but with large enough cohorts and carefully selected subgroups, common mechanisms should emerge. Finally, while many candidate risk genes have emerged for mental disorders, none appear specific for any single diagnosis. Neuregulin, disrupted-in-schizophrenia 1 (DISC1), and D-amino acid oxidase activator (DAOA; also known as G72), for instance, have been associated with both schizophrenia and bipolar disorder $(46,47)$. There may be a common set of risk genes for psychopathology, with the form of mental disorder determined by other factors.

\section{Mental disorders are not treated sufficiently with current medications}

Psychiatric medications are used widely for adults and children with mental disorders. As noted above, we have gone from firstgeneration to second-generation antidepressant and antipsychotic compounds. The second-generation compounds have fewer or different side effects, but with few exceptions, they do not seem to be more effective $(13,14)$. Nevertheless, these compounds are heavily used. In 2007, the third and fourth most heavily purchased medications in the United States were antipsychotics and antidepressants, respectively, with a combined market of $\$ 25$ billion (48). Remarkably, despite the heavy use of these medications, we have no evidence that the morbidity or mortality of mental disorders has dropped substantially in the past decades.

Comparative effectiveness studies have begun to examine the value of newer, expensive medications relative to older, less expen- 


\section{Table 1}

Are second-generation antipsychotic medications (olanzapine, quetiapine, risperidone, ziprasidone) more effective than first-generation antipsychotic medications (perphenazine, haloperidol, molindone)?

\section{Study name}

Clinical Antipsychotic

Trials of Intervention

Effectiveness (CATIE)

(49)

\section{Department of Veterans \\ Affairs Cooperative \\ Study Group on the \\ Cost-Effectiveness \\ of Olanzapine (50) \\ Cost Utility of the \\ Latest Antipsychotic \\ Drugs in Schizophrenia}

Study (CUtLASS 1)

(51)

$$
\begin{aligned}
& \text { Treatment of Early- } \\
& \text { Onset Schizophrenia } \\
& \text { Spectrum Disorders } \\
& \text { (TEOSS) study (52) }
\end{aligned}
$$

\section{Treatments}

Olanzapine, perphenazine, quetiapine, risperidone, or ziprasidone

Olanzapine or haloperidol

First- or second-generation antipsychotic medications

\begin{abstract}
Olanzapine, risperidone,
\end{abstract} or molindone

\section{Design}

1,493 patients with schizophrenia were randomly assigned to one of the drug treatments.

309 patients with schizophrenia or schizoaffective disorder were randomly assigned to one of the drug treatments.

227 patients with schizophrenia, schizoaffective disorder, or delusional disorder were randomly assigned to receive either a firstor second-generation antipsychotic drug treatment.

119 youths (aged 8-19 years) with schizophrenia, schizoaffective disorder, or schizophreniform disorder were randomly assigned to one of the drug treatments.

\begin{abstract}
Results
No differences in symptom improvement between the treatment conditions were observed; differences in side effects and likelihood of discontinuation were observed across drug treatments; olanzapine demonstrated the lowest rate of discontinuation but was associated with increased weight gain.
\end{abstract}

Olanzapine and haloperidol demonstrated equal effectiveness in compliance, symptoms, extrapyramidal symptoms, and overall quality of life; olanzapine was associated with increased weight gain and greater cost.

No differences in quality of life, symptoms, or associated costs of care between first-generation and second-generation antipsychotic drug treatment were found.

No differences in response rate or magnitude of symptom reduction between the treatment conditions were observed; olanzapine and risperidone were associated with increased weight gain, while molindone treatment was associated with a greater number of self-reports of akathisia. sive, and less commonly used compounds. For the antipsychotics, there are now four large-scale studies demonstrating the lack of superiority of the second-generation compounds over first-generation compounds (Table 1) (49-52). For the antidepressants, with either first-generation or second-generation medications, the rate of response continues to be slow and low. In the largest effectiveness study to date, with more than 4,000 patients with major depressive disorder in primary care and community settings, only $31 \%$ were in remission after 14 weeks of optimal treatment with the second-generation medication citalopram, a selective serotonin reuptake inhibitor (53). In most double-blind trials of antidepressants, the placebo response rate hovers around 30\% (54). Bipolar disorder is a particular challenge. While a fraction of patients respond to lithium, for too many, there is no medication that controls mood swings (55).

The unfortunate reality is that current medications help too few people to get better and very few people to get well. Most clinical trials have used acute statistical symptom improvement as an outcome, rather than assessment of the long-term functional improvements that would be desired for treatment of a chronic illness. The development of new medications based on marketable modifications of existing medications will not provide breakthroughs for most people with mental disorders. There is an urgent need for a third generation of medications far more effective than those that are available today. We will only develop this next generation of medications through an understanding of molecular targets. In this Review Series, Martinowich et al. describe how we can follow this approach to develop the next generation of medications for bipolar disorder by identifying key cell signaling pathways that can be targeted (3).

\section{Summary}

This overview began with reflections on the mistaken concepts of 30 years ago and suggested that many of our current assumptions may prove incorrect 30 years from now. The subsequent articles in this Review Series reveal many of the most exciting and potentially fruitful lines of inquiry that are transforming our understanding of several mental disorders. This is an extraordinary moment in the science of mental disorders. Indeed, it is difficult to find a precedent in medicine for what is beginning to happen in psychiatry. The intellectual basis of psychiatry is shifting, from reliance on psychological principles and theory to research findings and understanding the brain through neuroscience. The basis of diagnosis is likely to be completely reformulated to consider biological information, such as genetics and imaging data. And just as we no longer use psychoanalysis for peptic ulcer disease, treatments such as antibiotics and deep brain stimulation are likely to replace current medications as we learn more about the pathophysiology of disorders, such as one form of childhood-onset OCD and depression $(2,4)$. In a commencement speech given at Morehouse College in 1959, Martin Luther King Jr. reflected on the Rip Van Winkle story and the tragedy that this fictional character had slept through the most important revolution in American history (56). We are now at the forefront of a revolution in psychiatry, and one would be remiss to let it pass by unnoticed.

Address correspondence to: Thomas Insel, National Institute of Mental Health, 6001 Executive Blvd., Room 8235, MSC 9669, Bethesda, Maryland 20892-9669, USA. Phone: (301) 443-3673; Fax: (301) 443-2578; E-mail: tinsel@mail.nih.gov. 
1. Lewis, D.A., and Sweet, R.A. 2009. Schizophrenia from a neural circuitry perspective: advancing toward rational pharmacological therapies. J. Clin. Invest. 119:706-716.

2. Mayberg, H.S. 2009. Targeted electrode-based modulation of neural circuits for depression. J. Clin. Invest. 119:717-725.

3. Martinowich, K., Schloesser, R.J., and Manji, H.K. 2009. Bipolar disorder: from genes to behavior pathways. J. Clin. Invest. 119:726-736.

4. Kalra, S.K., and Swedo, S.E. 2009. Children with obsessive-compulsive disorder: are they just "little adults"? J. Clin. Invest. 119:737-746.

5. Levitt, P., and Campbell, D.B. 2009. The genetic and neurobiological compass points toward common signaling dysfunctions in autism spectrum disorders. J. Clin. Invest. 119:747-754.

6. WHO. 2002. The world health report 2002 - reducing risks, promoting healthy life. WHO. Geneva, Switzerland. http://www.who.int/whr/2002/en/.

7. Kessler, R.C., et al. 2003. The epidemiology of major depressive disorder: results from the National Comorbidity Survey Replication (NCS-R). JAMA 289:3095-3105.

8. Kessler, R.C., et al. 2005. Lifetime prevalence and age-of-onset distributions of DSM-IV disorders in the National Comorbidity Survey Replication. Arch. Gen. Psychiatry. 62:593-602.

9. Institute of Medicine, Committee on Pathophysiology \& Prevention of Adolescent \& Adult Suicide, and Board on Neuroscience and Behavioral Health. 2002. Reducing suicide: a national imperative. S.K. Goldsmith, T.C. Pellmar, A.M. Kleinman, and W.E. Bunney, editors. National Academies Press. Washington, DC, USA. 496 pp.

10. Kung, H.C., Hoyert, D.L., Xu, J., and Murphy, S.L. 2008. Deaths: final data for 2005. Natl. Vital Stat. Rep. 56:1-120.

11. Colton, C.W., and Manderscheid, R.W. 2006. Congruencies in increased mortality rates, years of potential life lost, and causes of death among public mental health clients in eight states. Prev. Chronic Dis. 3:A42.

12. Lasser, K., et al. 2000. Smoking and mental illness: a population-based prevalence study. JAMA. 284:2606-2610.

13. Thomas, P. 2007. The stable patient with schizophrenia - from antipsychotic effectiveness to adherence. Eur. Neuropsychopharmacol. 17(Suppl. 2):S115-S122.

14. Cipriani, A., et al. 2005. Fluoxetine versus other types of pharmacotherapy for depression. Cochrane Database Syst Rev. CD004185.

15. Butler, A.C., Chapman, J.E., Forman, E.M., and Beck, A.T. 2006. The empirical status of cognitivebehavioral therapy: a review of meta-analyses. Clin. Psychol. Rev. 26:17-31.

16. Shean, G.D. 2007. Recent developments in psychosocial treatments for schizophrenic patients. Expert Rev. Neurother. 7:817-827.

17. Regier, D.A., et al. 1990. Comorbidity of mental disorders with alcohol and other drug abuse. Results from the Epidemiologic Catchment Area (ECA) Study. JAMA. 264:2511-2518.

18. Insel, T.R., and Quirion, R. 2005. Psychiatry as a clinical neuroscience discipline. JAMA. 294:2221-2224.

19. Bush, G. 1990. Presidential proclamation 6158. Library of Congress. http://www.loc.gov/loc/brain/ proclaim.html.

20. Meyer-Lindenberg, A.S., et al. 2005. Regionally specific disturbance of dorsolateral prefrontalhippocampal functional connectivity in schizophrenia. Arch. Gen. Psychiatry. 62:379-386.

21. Ressler, K.J., and Mayberg, H.S. 2007. Targeting abnormal neural circuits in mood and anxiety disorders: from the laboratory to the clinic. Nat. Neu- rosci. 10:1116-1124.

22. Graybiel, A.M., and Rauch, S.L. 2000. Toward a neurobiology of obsessive-compulsive disorder. Neuron. 28:343-347.

23. Rauch, S.L., Shin, L.M., and Phelps, E.A. 2006. Neurocircuitry models of posttraumatic stress disorder and extinction: human neuroimaging research - past, present, and future. Biol. Psychiatry. 60:376-382.

24. Shaw, P., et al. 2007. Attention-deficit/hyperactivity disorder is characterized by a delay in cortical maturation. Proc. Natl. Acad. Sci. U. S. A. 104:19649-19654.

25. Kandel, E.R., Schwartz, J.H., and Jessell, T.M. 2000. Principles of neural science. McGraw-Hill. New York, New York, USA. 1414 pp.

26. Wang, P.S., et al. 2005. Failure and delay in initial treatment contact after first onset of mental disorders in the National Comorbidity Survey Replication. Arch. Gen. Psychiatry. 62:603-613.

27. Warner, V., Mufson, L., and Weissman, M.M. 1995. Offspring at high and low risk for depression and anxiety: mechanisms of psychiatric disorder. J. Am. Acad. Child Adolesc. Psychiatry. 34:786-797.

28. Weissman, M.M., Warner, V., Wickramaratne, P., Moreau, D., and Olfson, M. 1997. Offspring of depressed parents. 10 years later. Arch. Gen. Psychiatry. 54:932-940.

29. Kessler, R.C., and Walters, E.E. 1998. Epidemiology of DSM-III-R major depression and minor depression among adolescents and young adults in the National Comorbidity Survey. Depress. Anxiety. 7:3-14.

30. Cannon, T.D., et al. 2008. Prediction of psychosis in youth at high clinical risk: a multisite longitudinal study in North America. Arch. Gen. Psychiatry. 65:28-37.

31. Sullivan, M., et al. 2007. Response to joint attention in toddlers at risk for autism spectrum disorder: a prospective study. J. Autism Dev. Disord. 37:37-48.

32. Haberland, C. 2007. Clinical neuropathology: text and color atlas. Demos Medical Publishing. New York, New York, USA. 324 pp.

33. Aminoff, M.J. 2001. Neurology and general medicine. Churchill Livingstone. New York, New York, USA. 1147 pp.

34. Klein, R.J., et al. 2005. Complement factor $\mathrm{H}$ polymorphism in age-related macular degeneration. Science. 308:385-389.

35. Duerr, R.H., et al. 2006. A genome-wide association study identifies IL23R as an inflammatory bowel disease gene. Science. 314:1461-1463.

36. Saxena, R., et al. 2007. Genome-wide association analysis identifies loci for type 2 diabetes and triglyceride levels. Science. 316:1331-1336.

37. Scott, L.J., et al. 2007. A genome-wide association study of type 2 diabetes in Finns detects multiple susceptibility variants. Science. 316:1341-1345.

38. Zeggini, E., et al. 2007. Replication of genome-wide association signals in UK samples reveals risk loci for type 2 diabetes. Science. 316:1336-1341.

39. Sklar, P., et al. 2008. Whole-genome association study of bipolar disorder. Mol. Psychiatry. 13:558-569.

40. O'Donovan, M.C., et al. 2008. Identification of loci associated with schizophrenia by genome-wide association and follow-up. Nat. Genet. 40:1053-1055.

41. Szatmari, P., et al. 2007. Mapping autism risk loci using genetic linkage and chromosomal rearrangements. Nat. Genet. 39:319-328.

42. Xu, B., et al. 2008. Strong association of de novo copy number mutations with sporadic schizophrenia. Nat. Genet. 40:880-885.

43. Sebat, J., et al. 2007. Strong association of de novo copy number mutations with autism. Science. 316:445-449.

44. Walsh, T., et al. 2008. Rare structural variants dis- rupt multiple genes in neurodevelopmental pathways in schizophrenia. Science. 320:539-543.

45. Stefansson, H., et al. 2008. Large recurrent microdeletions associated with schizophrenia. Nature. 455:232-236

46. Maier, W. 2008. Common risk genes for affective and schizophrenic psychoses. Eur. Arch. Psychiatry Clin. Neurosci. 258(Suppl. 2):37-40

47. Chubb, J.E., Bradshaw, N.J., Soares, D.C., Porteous, D.J., and Millar, J.K. 2008. The DISC locus in psychiatric illness. Mol. Psychiatry. 13:36-64.

48. IMS Health Inc. 2007. IMS national sales perspectives 2007. http://www.imshealth.com.

49. Lieberman, J.A., et al. 2005. Effectiveness of antipsychotic drugs in patients with chronic schizophrenia. N. Engl. J. Med. 353:1209-1223.

50. Rosenheck, R., et al. 2003. Effectiveness and cost of olanzapine and haloperidol in the treatment of schizophrenia: a randomized controlled trial. JAMA. 290:2693-2702.

51. Jones, P.B., et al. 2006. Randomized controlled trial of the effect on Quality of Life of second- vs firstgeneration antipsychotic drugs in schizophrenia: Cost Utility of the Latest Antipsychotic Drugs in Schizophrenia Study (CUtLASS 1). Arch. Gen. Psychiatry. 63:1079-1087.

52. Sikich, L., et al. 2008. Double-blind comparison of first- and second-generation antipsychotics in early-onset schizophrenia and schizoaffective disorder: findings from the Treatment of Early-Onset Schizophrenia Spectrum Disorders (TEOSS) study. Am. J. Psychiatry. 165:1420-1431.

53. Rush, A.J. 2007. STAR*D: what have we learned? Am. J. Psychiatry. 164:201-204.

54. Walsh, B.T., Seidman, S.N., Sysko, R., and Gould, M. 2002. Placebo response in studies of major depression: variable, substantial, and growing. JAMA. 287:1840-1847.

55. Smith, L.A., Cornelius, V., Warnock, A., Bell, A., and Young, A.H. 2007. Effectiveness of mood stabilizers and antipsychotics in the maintenance phase of bipolar disorder: a systematic review of randomized controlled trials. Bipolar Disord. 9:394-412.

56. King, M.L., Carson, C., and Holloran, P. 1998. A knock at midnight: inspiration from the great sermons of Reverend Martin Luther King, Jr. Warner Books. New York, New York, USA. 256 pp.

57. Halme, L., et al. 2006. Family and twin studies in inflammatory bowel disease. World J. Gastroenterol. 12:3668-3672.

58. Selby,J.V., et al. 1991. Concordance for dyslipidemic hypertension in male twins. JAMA. 265:2079-2084.

59. Hyttinen, V., Kaprio, J., Kinnunen, L., Koskenvuo, M., and Tuomilehto, J. 2003. Genetic liability of type 1 diabetes and the onset age among 22,650 young Finnish twin pairs: a nationwide follow-up study. Diabetes. 52:1052-1055.

60. Seddon, J.M., Cote, J., Page, W.F., Aggen, S.H., and Neale, M.C. 2005. The US twin study of age-related macular degeneration: relative roles of genetic and environmental influences. Arch. Ophthalmol. 123:321-327.

61. Kendler, K.S., and Prescott, C.A. 1999. A population-based twin study of lifetime major depression in men and women. Arch. Gen. Psychiatry. 56:39-44.

62. Cardno, A.G., and Gottesman, I.I. 2000. Twin studies of schizophrenia: from bow-and-arrow concordances to star wars $\mathrm{Mx}$ and functional genomics. Am. J. Med. Genet. 97:12-17.

63. Kieseppa, T., Partonen, T., Haukka, J., Kaprio, J., and Lonnqvist, J. 2004. High concordance of bipolar I disorder in a nationwide sample of twins. Am. J. Psychiatry. 161:1814-1821.

64. Folstein, S.E., and Rosen-Sheidley, B. 2001. Genetics of autism: complex aetiology for a heterogeneous disorder. Nat. Rev. Genet. 2:943-955. 